(REVIEW ARTICLE)

\title{
Understanding and addressing eating disorders
}

\author{
Popova Aneta Tincheva ${ }^{1, *}$ \\ Department of Catering and tourism, University of food technologies, 26 Maritsa blvd., 4000, Plovdiv, Bulgaria.
}

Publication history: Received on 28 April 2019; revised on 14 May 2019; accepted on 16 May 2019

Article DOI: https://doi.org/10.30574/wjarr.2019.2.1.0032

\begin{abstract}
Eating disorders include conditions such as excessive and unhealthy reductions in food intake or overeating, distress, and overuse of body shape and weight. Some of the most recognizable among society are anorexia nervosa, bulimia nervosa, binge eating and orthorexia. Eating disorders are very often diagnosed at a late stage of the disease and curability is quite difficult. A multidisciplinary approach and lifelong efforts are needed in order to address such conditions. The psychology of eating disorders has been widely studied over the years. However, very few guidelines address the re-feeding process of the people involved. This review focuses on the nutritional point of view of eating disorders and can be a valuable addition to treatment of these serious conditions.
\end{abstract}

Keywords: Eating disorders; Nutritional deficiencies; Meal plans; Culinary techniques

\section{Introduction}

Unnatural eating habits lead to conditions called eating disorders. They harm both the physical and mental health. Feeding is controlled by many factors such as appetite, food availability, family habits, cultural features, personal will, etc. Adequate nutritional practices are manifested with the proper intake of proteins, carbohydrates, fats, minerals and elements. Eating disorders include conditions such as excessive and unhealthy reductions in food intake or overeating, distress, and overuse of body shape and weight.

Eating disorders affect more women, although the number of diagnosed men is not so small [1]. Food-related disorders are the leading cause of death for women under the age of 30 . There is not a single factor leading to eating disorders, but multifactorial-complex interactions between biological issues such as genetics and metabolism; psychological problems such as control, coping skills, personality factors; family issues as well as culture influence these conditions.

Nutritional disorders are, by definition, mental disorders that are predominantly in the form of unusual dietary behavior [2]. Disturbed eating behavior can be characterized by different patterns i.e. restriction conditions or overeating. The diagnosis is based on the following aspects: body weight relative to height, model of nutritional behavior, weight control methods that are used, perception and assessment of the body, as well as the physical consequences of severely reduced body weight.

The Renfrew Center Foundation's statistics show that up to 70 million people worldwide have some type of eating disorder, half of which meet the criteria for depression. Only in the United States, people with nutritional disturbances are about 24 million [3]. The same statistics indicate that only $10 \%$ receive treatment. Mortality in anorexia nervosa is up to $20 \%$ due to complications resulting from the disease, as well as suicide. According to the National Institute of Mental Health in the United States, about 3\% of adolescents are affected by eating disorders and the majority of them are not receiving treatment. Data shows that about $0.3 \%$ of the population is diagnosed with anorexia, $0.9 \%$ with bulimia and $1.6 \%$ with a tendency to overeat [4]. The eating disorder may last for more than 10 years, with nearly 80

\footnotetext{
${ }^{*}$ Corresponding author

E-mail address: popova_aneta@yahoo.com
} 
percent of the patients recovering. Although eating disorders are the highest mortality among mental disorders, mortality data are different. Franko et al [5] report a 4\% mortality rate in anorexia nervosa and 3.9\% - in bulimia.

The aim of this review was to determine the most common types of eating disorders and to pinpoint the necessity of adequate nutritional data concerning the treatment of such health problems.

\section{Common types of eating disorders}

\subsection{Anorexia nervosa}

Anorexia is characterized by at least $15 \%$ lower than normal weight for the appropriate height and age that is selfinduced through fasting or severely restricted eating [6]. It may be a cause of excessive physical exercise, diuretic or laxative medication or self-induced vomiting. There is a strong persistent fear of weight gain. People with anorexia may feel abdominal swelling even after inducing small amounts of food. They often feel cold, are constantly tired, apathetic, and may have constipation, stomachache and other illness symptoms. Anorexia may be a cause of disturbances and slowing of development during puberty, the occurrence of osteoporosis, damage to the heart, liver, and kidneys. People with anorexia usually lose interest in all social contacts and are often depressed.

\subsection{Bulimia nervosa}

Bulimia is a serious eating disorder, which is expressed in frequent consumption of large amounts of food, followed by purposeful "cleansing of the organism" (induced vomiting, diuretic intake, etc.) [7]. Bulimia is associated with loss of control over nutrition and a constant, extreme concentration on body weight [8]. This eating disorder, similar to anorexia, occurs exclusively in women, most often between the ages of 16 and 40 . Bulimia has many causes, but one of the most common is depression. Another trigger point is overweight or obesity in the past, especially in childhood and puberty, which creates special overweight control dominance. There are women with anorexia who have bulimic episodes.

\subsection{Binge eating}

Binge eating disorder include is associated recurrent episodes of binge eating. Binge eaters eat much more rapidly than normal, until they feel uncomfortably full. No compensatory behavior is observed as individuals with bulimia [9] but binge eaters are more concerned about their weight and body shape when compared to those who are only obese [10]. Binge eating disorder might be influenced by several factors (body image, depression, anxiety, impulsivity and low selfesteem) [11]; therefore, it requires an evaluation that takes into account different aspects involving its origin and maintenance [12].

\subsection{Orthorexia}

Orthorexia is described as a pathological preoccupation with eating only healthy food [13]. Unlike bulimia and anorexia, which are related to the quantities of food intake, orthorexia addresses food quality obsession. The word orthorexia originates from the Greek word ortho, which means accurate and orexi translated as appetite. According to the DSM-5 [2], orthorexia would be most appropriately categorized as a distinct subtype of "avoidant/restrictive food intake disorder". Orthorexics have a strong guilt if they eat something forbidden. Foods containing salt, fat, coloring, nitrate, preservatives, sugar are categorized as forbidden/unhealthy. Dunn \& Bratman [13] propose the following diagnostic criteria for orthorexia obsessive focus on healthy eating, compulsive bahaviour, self-imposed dietary rules, and escalating restrictions related to eliminating certain food groups, weight loss, etc. This pathological condition can lead to serious health problems.

\section{Understanding nutritional deficiencies}

Both food restriction and unhealthy excessive eating can lead to various nutritional deficiencies. For instance, the lack of fat in the body prevents the absorption of fat-soluble vitamins such as A, D, E, K. Without meat, blood iron levels decrease significantly. Even if iron and protein-rich foods of plant origin are eaten, they are of lower quality. The lack of some of the essential nutrients in the body can lead to anemia, lack of vitamins and energy.

Mineral deficiency treatment depends on the type and severity of the deficiency. Concomitant diseases are also a factor. A physician may arrange additional studies to identify the amount of damage incurred before deciding on a treatment plan. This might include treatment of other illnesses or a change in treatment. Changing the eating habits can be effective 
if there is a small mineral deficiency. People with anemia due to lack of iron in food can be advised to eat more meat, poultry, eggs, and iron-enriched cereals. If the deficit is more severe, a nutritionist consult is advisory, where guidelines will be given on how to eat a well-balanced diet rich in fruits, vegetables and whole grains.

Some mineral flaws cannot be treated with just a diet. The affected may need to take appropriate supplements alone or in combinations that help the body absorb or use the mineral. Vitamin D, for example, is usually taken together with calcium. Calcium is the most abundant mineral found in the human body. More than $99 \%$ of the total body calcium is stored in the bones and teeth. The remaining $1 \%$ is found in the blood, muscles and intracellular fluid. Calcium is needed for muscle contraction, blood vessel constriction and relaxation, hormone secretion, enzymes, and signaling nervous system [14]. A constant level of calcium is maintained in body fluids and tissues so that these life processes of the body function effectively. The body gets the calcium it needs in two ways: through dietary intake of calcium-rich foods, or by extracting it from the bone, which leads to bone structure weakening. This happens when the levels of calcium in the blood are too low [15].

Copper is involved in many biochemical reactions in human cells. Copper is a constituent of multiple enzymes. It is responsible for many reactions in the body i.e. regulating cell metabolism, tissue formation, absorption, storage, and metabolism of iron. Clinically, in the absence of copper, the following symptoms occur: headache, fatigue, fatigue, weakness, dizziness can be observed, which are all signs of anemia with leukocyte deficiency [16].Iodine is an element required for the synthesis of thyroid hormones. Chronic iodine deficiency can lead to a variety of health problems, including thyroid dysfunction and various neurological, gastrointestinal and skin abnormalities. Deficiencies are characterized with difficulties to memorize, listen to information, to concentrate, etc. The metabolism slows down [17]. The skin is drier and wrinkles start to appear. Intracranial pressure is impaired and headaches constantly occur [18].

Iron is a major mineral and an important component of proteins involved in the transport of oxygen and metabolism. Iron is also an important cofactor in neurotransmitters synthesis such as dopamine, norepinephrine and serotonin [19]. Iron deficiency is the most common and widespread eating disorder worldwide.Phosphorus is a vital mineral for the formation of bones and teeth, and healthy bones and soft tissues require calcium and phosphorus to grow and develop throughout life [20]. The deficiency of phosphorus in the body has a pronounced negative impact on the health of the bone system. Even if sufficient calcium is taken if it is not accompanied by adequate phosphorus intake, hydroxyapatite crystals cannot be formed [21]. In this way, there is a risk of lowering the bone density and the development of osteoporosis.

Magnesium plays an important role in muscle contractions by regulating cellular level of neuromuscular excitability. Like vitamin D, magnesium helps calcium and phosphorus to settle in bone tissue. Takes part in the formation of cartilage and collagen (an important building block protein) [22].Vitamin A is a fat-soluble vitamin that is needed for a large number of biological processes. Vitamin A (fig. 1) is a group of unsaturated nutritional organic compounds including retinol, retinal, retinoic acid, and several pro vitamin A carotenoids. A major biological function of vitamin A (such as the retinal metabolite) is in the retinoid cycle [23]. Prolonged deficiency can lead to xerophthalmia (dry eyes) and ultimately to night blindness or total blindness, as well as skin diseases, infections (such as measles), diarrhea and respiratory diseases [24].

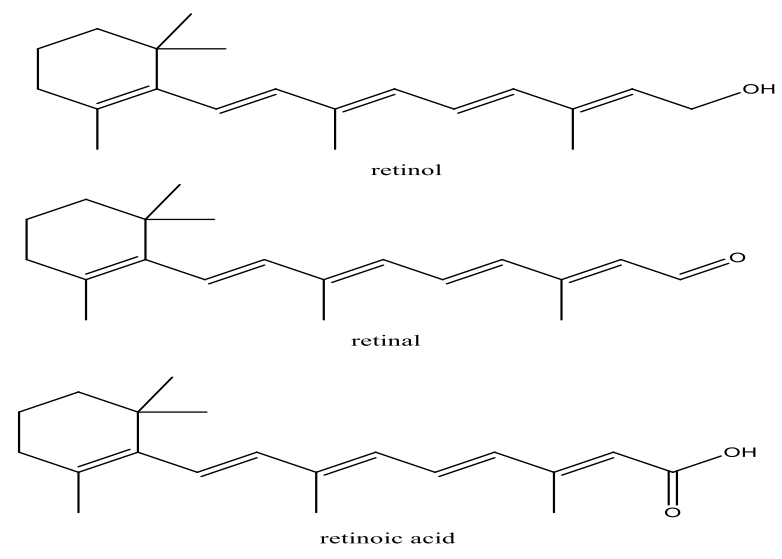

Figure 1 Vitamin A compounds 
Thiamine is a water-soluble vitamin B complex which participates in numerous functions of the body, including the nervous system and muscles functioning; flow of electrolytes into and from nerve and muscle cells (via ion channels); multiple enzyme processes (via coenzyme thiamine pyrophosphate); carbohydrate metabolism; and production of hydrochloric acid (which is necessary for proper digestion) [25]. Riboflavin is a water-soluble vitamin that plays a vital role in metabolic processes in the body and is necessary for normal cell function, growth and energy production [26].

Pantothenic acid is essential for all living beings and is a component of CoA, a molecule that is necessary for multiple vital chemical reactions to occur in cells. Pantothenic acid is essential for the metabolism of carbohydrates, proteins and fats, as well as for the synthesis of hormones and cholesterol [27]. Pantothenic acid deficiency is very rarely seen in humans, except for severe malnutrition.Pyridoxine is needed for the synthesis of serotonin and norepinephrine neurotransmitters as well as for the formation of myelin [28]. Pyridoxine deficiency in adults primarily affects the peripheral nerves, skin, mucous membranes, and the blood cell system.

Vitamin $\mathrm{B}_{12}$ is an essential water-soluble vitamin, which helps maintain healthy nerve cells and red blood cells [29]. Studies have shown that vitamin $B_{12}$ deficiency can lead to abnormal neurological and psychiatric symptoms. The watersoluble ascorbic acid aid in the formation of collagen in bones, cartilage, muscles and blood vessels and absorption of iron in the body [30]. The main biological function of vitamin $\mathrm{D}$ is to maintain normal levels of calcium and phosphorus in the blood (fig. 2).

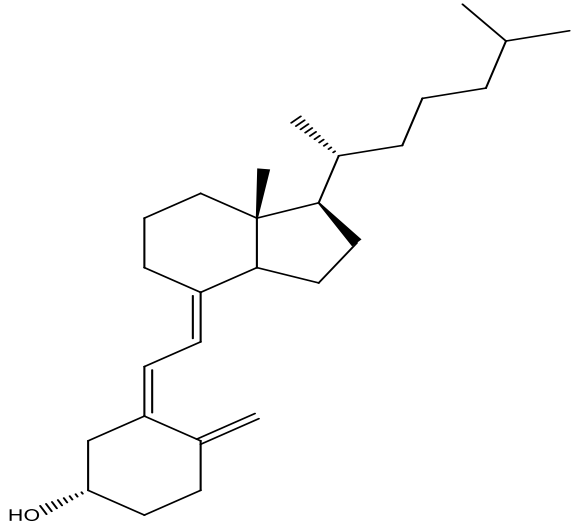

cholecalciferol

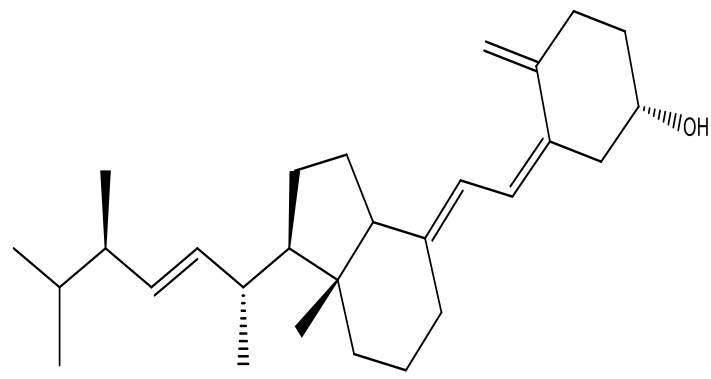

Ergocalciferol

Figure 2 Forms of vitamin $\mathrm{D}\left(\mathrm{D}_{2}\right.$ - ergocalciferol and $\mathrm{D}_{3}$ - cholecalciferol)

Vitamin D helps in the absorption of calcium, which helps to create and maintain healthy bones [31].

Folic acid is a form of water-soluble vitamin A. It is often used in combination with other vitamins from the group of vitamin B formulations. Deficiency of folic acid will occur if the body does not receive enough of the folic acid from the diet. Folic acid has been shown to be effective in the treatment of anemia due to folate deficiency [32]. Folic acid deficiency causes megaloblastic anemia. Patients at risk should receive adequate amounts of thiamine and magnesium.

Thiamine and magnesium levels are often low in starvation [33]. A vitamin/mineral supplement is often given at the start of the re-feeding. Many malnourished people suffer from a lack of vitamin D and calcium. Numerous reports have documented low levels of zinc anorexia and bulimia [34]. Often, people suffering from eating disorders are anemic due to iron and folic acid deficiency [35].

Several food groups are sources of vitamins and minerals. Table 1 is a visual presentation of recommended natural sources of nutrients 
Table 1 Nutrient content in some foods [36]

\begin{tabular}{|c|c|c|c|}
\hline Source & Vitamins & Minerals & $\begin{array}{l}\text { Other } \\
\text { nutrients }\end{array}$ \\
\hline Meat & $\mathrm{B}_{6}, \mathrm{D}$ & $\mathrm{Mg}, \mathrm{Fe}, \mathrm{Ca}$ & animal protein \\
\hline Eggs & $\mathrm{A}, \mathrm{D}, \mathrm{B}_{12}$ & $\mathrm{Na}, \mathrm{K}, \mathrm{Ca}, \mathrm{Fe}, \mathrm{Mg}$ & animal protein \\
\hline Dairy products (milk, yoghurt, cheese) & A, D & $\mathrm{Na}, \mathrm{K}, \mathrm{Ca}$ & animal protein \\
\hline Grains (wheat, barley, spelt, corn) & $\mathrm{A}, \mathrm{B}_{1}, \mathrm{~B}_{3}, \mathrm{~B}_{5}$ & Fe, P, K, Zn & plant protein, carotene \\
\hline (quinoa, amaranth, & A & $\mathrm{P}, \mathrm{Fe}, \mathrm{Mg}, \mathrm{Zn}$ & $\begin{array}{l}\text { plant protein, fiber, } \\
\text { omega fats }\end{array}$ \\
\hline Nuts (almonds, hazelnuts, cashew, walnuts) & A & $\mathrm{K}, \mathrm{Ca}, \mathrm{Fe}, \mathrm{Mg}$ & fiber, fats \\
\hline Seeds (sesame, poppy, flaxseed, pumpkin) & $\mathrm{C}, \mathrm{B}_{6}$ & $\mathrm{Na}, \mathrm{K}, \mathrm{Ca}, \mathrm{Fe}, \mathrm{Mg}$ & plant protein \\
\hline Tubes (carrots, potato, manioc, yam) & $\mathrm{B}_{6}, \mathrm{~K}$ & $\mathrm{~K}$ & fiber, carotene \\
\hline Nightshades (tomato, eggplant, pepper) & $\mathrm{A}, \mathrm{C}$ & $\mathrm{Mg}, \mathrm{K}, \mathrm{Ca}$ & fiber \\
\hline
\end{tabular}

\section{Appropriate meal planning}

In the event of malnutrition, the diet plan involves regular small meals and supportive snacks, easy to eat and digest to make sure the menu is not too tiring or stressful. After about a week, under such a regime, the body will begin to recover, as the body temperature will normalize and the body will have energy. The next stage involves the construction of new, healthy tissues. Initially, a malnourished person will have lower-than-expected energy needs of 1500-2000kcal per day.(In order to maintain an approximate weight gain of $500 \mathrm{~g} / \mathrm{week}$.) Within a few weeks, the intake should be increased to about $3000 \mathrm{kcal}$ or more to fully recover and return to full physical health. As people work to achieve their target weights, realistic goals are set at $0.5-1 \mathrm{~kg} /$ week. Nutritionists help with meal choices and provide a structured eating plan that ensures nutritional adequacy and inclusion of all major food groups. It is believed that the amount of solid food should not exceed the amount that the particular person would normally eat. Possible food allergies and religious and cultural practices are also taken into account when building a nutritional plan. Daily intake usually starts at 30-40 kcal/kg per day. During the weight gain phase, it may need to be gradually increased to $70-100 \mathrm{kcal} / \mathrm{kg}$ per day for some people i.e. men because they need a much higher number of calories to gain weight. It should be borne in mind that delayed function of the digestive tract reduces the person's ability to tolerate 1000 calories/day. In such circumstances, it is often more effective to start with 200-300 calories over the usual caloric intake (for example, a person that consumes 400 calories a day may need to start at 600-700 calories a day). In addition to increasing calorie intake, vitamin and mineral supplements should be introduced to the diet. Serum potassium levels should be monitored regularly in people who are systemically vomiting. Hypocalcemia should be treated with oral or intravenous potassium intake and rehydration.

Physical activity should be adapted to food intake, taking into account bone mineral density and cardiac function. For seriously underweight people, exercise should be limited and always carefully monitored. After weight gain, changes in mood-related and worrying symptoms, as well as in the physical condition, can be expected. Initially, re-feeding may be associated with mild fluid retention, and patients who suddenly stop taking laxatives or diuretics may experience fluid retention for several weeks, often accompanied by abdominal swelling.

In the event of excessive food intake, there are various regimes which can be applied. Very low calorie diets (VLCD) are hypo-energy diets that deliver the body up to $800 \mathrm{kcal}$ per day. Used since the 1920s, they are most often offered as liquid formulations with different macronutrient content and provide rapid weight loss. They are suitable for use by people with body mass index (BMI) $\geq 30 \mathrm{~kg} / \mathrm{m}^{2}$ suffering from concomitant diseases that may be affected by abrupt weight loss. Expected results range from $2-3 \mathrm{~kg}$ per week to $12 \mathrm{~kg}$ over a 12 -week period. VLCDs should not be used for long periods of time, or applied to children, pregnant or lactating women, people suffering from cardiovascular, renal diseases, osteoporosis, etc. They should only be used under strict medical supervision.

Standard low calorie diets are diets high in carbohydrates (55-60\% of total energy), low fat $(<30 \%)$, moderate protein content (15-20\%), providing a negative energy balance (500 - $1000 \mathrm{kcal}$ under normal energy requirements). They are rich in complex carbohydrates and fibers and have a low glycemic index. These diets may result in a $10 \%$ weight loss 
over a period of 6 months, while significantly reducing the risk of developing concomitant diseases. They do not pose any risk to human health and are recommended by health authorities around the world.

Low Carbohydrate Diets are diets with extremely low carbohydrate, high fat and protein content. The physiological explanation of their potential efficacy is maintaining relatively low insulin levels that would favor weight loss.

Low-fat diets are designed to prevent cardiovascular disease. These diets containing 10-15\% fat are gradually gaining popularity. Their effect on body weight loss is not particularly different from standard low calorie diets. At the same time, fat-rich diets contain a large amount of fiber and may prevent the absorption of certain trace elements and fatsoluble vitamins, so they are not recommended for long-term use.

High protein diets contain a relatively high amount of proteins (about 25\%). Since proteins have a pronounced thermogenic effect and prolong the feeling of satiety, high protein diets have been greeted with great hopes. So far, however, there is no evidence that they stimulate body weight loss. In addition, the intake of protein foods rich in purines (red meat and seafood) hides some risk of developing gout and kidney disease.

\section{Cooking techniques}

Contemporary nutritional science highly appreciates every culinary method that maximizes food's nutritional value. During pre-treatment (washing, cleaning, cutting, etc.) all standard hygiene requirements should be applied [37]. Fruits and vegetables should preferably be eaten raw, in their natural form - uncut or as salads (to prevent loss of nutrients). Meat and fish are thawed slowly, at room temperature rather than in hot water or oven. Frozen vegetable products are placed directly into hot water without defrosting in advance. The microbiological safety of food is achieved through appropriate heat treatment. The applied heat treatment methods should be sparing and maximally preserving food's beneficial ingredients. For this purpose, steam cooking, sous-vide and baking are quite appropriate. Vegetables should be placed in boiling water covered with a lid. When placed in cold water, enzymes destroy much of the vitamin, especially vitamin C. Frying should be avoided because it produces oxidized fat products that are toxic and irritate the lining of the gastrointestinal tract [38]. Under the influence of heat treatment, there are significant changes in food products: cell walls are destroyed, proteins are denatured, starch is gelatinized, and carbohydrates are caramelized, making the product milder and softer and the digestive enzymes more accessible for digestion.

The body does not only not absorb hemicellulose and protoptectin, which make up the cell wall of products of plant origin, but also prevent the absorption of other nutrients found in plant cells [39]. Upon heating, the hemicellulose is partially degraded and the protopetin switches to pectin, which is well absorbed by the body.

The taste and nutritional value of the food depends largely on the heat treatment method. When cooked, water-soluble proteins, fats, carbohydrates, mineral salts, vitamins, nitrogen substances are extracted from the product and passed into the water [40]. Loss of nutrients depends on the cooking conditions: when the meat is dipped in cold water (especially if the product is finely chopped), a maximum of nutrients transfer to the broth. As a result, concentrated broths with high nutritional value and taste qualities are obtained. They have a stimulating effect on the secretory function of the digestive glands. To limit carbohydrates, besides excluding them from daily meals, it is recommended to soak and then cook starch-containing foods [41]. To reduce the introduction of calcium salts in the body, milk and dairy products, green leaves, some varieties of apples, etc. should be excluded from the diet [42].

\section{Ethical issues when dealing with eating disorders}

Ethical dilemmas cover many aspects of the treatment of people with eating disorders.

Ethical issues are often related to the following issues:

- In which cases it is ethical to withdraw the right to a decision and force the person to be hospitalized or be compelled to eat;

- In which cases the person can make adequate decisions and how this competence is being determined;

- How ethical is it to take away personal freedom (for example, monitoring food intake and bathroom usage, limiting acquaintances, etc.);

- To what extent is forceful treatment effective? 
Such ethical dilemmas are of great importance in the eating disorders treatment. Respect for the autonomous decisions of others is of utmost importance. In many cases, in order to improve the physical condition of a person, decisions contrary to ethical norms are made. The nature of eating disorders is vague and complex, so there are no clear, universal and best decisions. In fact, decisions other than ethical expectations can be excellent depending on the circumstances and the context of each situation [43].

In the treatment of children and adolescents with eating disorders, there are unique challenges in the ethical decisionmaking. There is a desire to provide the opportunity for minors to make their own treatment decisions.

In order to distinguish the best course of action, a comprehensive health risk assessment, consideration of all treatment options, evidence of the potential efficacy of each of these options, and the acceptability of the options for the patient should be considered [43].

\section{Conclusion}

Eating disorders are included in DSM-IV, DSM-V in order to improve diagnoses, treatment, and research. They are generally associated with negative self-evaluations and episodes of abnormal food intake. All eating disorders are generally considered curable when diagnosed at their early stages. Early detection of abnormal eating habits can lead to the prevention of these serious conditions.

\section{Compliance with ethical standards}

\section{Disclosure of conflict of interest}

Declared none

\section{References}

[1] Herpertz S, Hagenah U, Vocks S, Von Wietersheim J, Cuntz U and Zeeck A. (2011). The diagnosis and treatment of eating disorders, German Society of Psychosomatic Medicine and Psychotherapy; German College for Psychosomatic Medicine. Deutsches Ärzteblatt International, 108(40), 678-85.

[2] The American Psychiatric Association. (2013). Diagnostic and Statistical Manual of Mental Disorders (DSM-5), American Psychiatric Publishing.

[3] Kessler C, Avenevoli S, Costello J, Green G, Gruber J, Heeringa S, Merikangas R, Pennell E, Sampson A and Zaslavsky M. (2009). Design and field procedures in the US National Comorbidity Survey Replication Adolescent Supplement (NCS-A), International Journal of Methods in Psychiatric Research, 18(2), 69-83.

[4] Le Grange D, Swanson A, Crow J and Merikangas R. (2012). Eating disorder not otherwise specified presentation in the US population. International Journal of Eating Disorders, 45(5), 711-718.

[5] Franko L, Keshaviah A, Eddy T, Krishna M, Davis C, Keel K and Herzog B. (2013). A longitudinal investigation of mortality in anorexia nervosa and bulimia nervosa. American Journal of Psychiatry, 170(8), 917-25.

[6] Pearce S and Morton R. (2004). Origins of Anorexia nervosa. Eur. Neurol., 52, 191-192.

[7] Gross J, Rosen C, Leitenberg H and Willmuth E. (1986). Validity of the Eating Attitudes Test and the Eating Disorders Inventory in bulimia nervosa. Journal of Consulting and Clinical Psychology, 54(6), 875-876.

[8] American Psychiatric Association Work Group on Eating Disorders. (2000). Practice guideline for the treatment of patients with eating disorders (revision). American Journal of Psychiatry, 157(1 Suppl.), 1-39.

[9] Vigo E, Castro N, Dorpinghaus A, Weidema H, Cardinali P and Siri N. (2007). Nonlinear analysis of heart rate variability in patients with eating disorders. World Journal of Biological Psychiatry, 11, 17.

[10] Didie R and Fitzgibbon M. (2005). Binge eating and psychological distress: is the degree of obesity a factor? Eating Behaviors, 6, 35-41.

[11] Malevani J, Mobascher J, Cordes J and Mobascher A. (2007). Differential diagnosis of coagulation abnormalities in borderline personality disorder. World Journal of Biological Psychiatry, 10, 13. 
[12] Mazzeo E, Saunders R and MitcMhell S. (2006). Gender and binge eating among bariatric surgery candidates. Eating Behaviors, 7, 47-52.

[13] Dunn T. and Bratman S. (2016). On orthorexia nervosa: A review of the literature and proposed diagnostic criteria. Eating behaviours, 21, 11-17.

[14] Kelly C and Wilkins R. (2004). Treatment of Benign Bone Lesions with an Injectable Calcium Sulfate-Based Bone Graft Substitute. Orthopedics, 27, 131-135.

[15] Darling L, Millward J, Torgerson J, Hewitt E and Lanham-New A. (2009). Dietary protein and bone health: a systematic review and meta-analysis. American Journal of Clinical Nutrition, 90(6), 1674-1692.

[16] Wazir M and Ghobrial I. (2017). Copper deficiency, a new triad: anemia, leucopenia, and myeloneuropathy. Journal of Community Hospital Internal Medicine Perspectives, 7(4), 265-268.

[17] Zimmermann M. and Boelaert K. (2015). Iodine deficiency and thyroid disorders. Diabetes and Endocrinology, $3(4), 286-295$.

[18] Gunnarsdottir I. and Dahl L. (2012). Iodine intake in human nutrition: a systematic literature review. Food \& Nutrition Research, 56(1), 19731.

[19] Pain D and Dancis A. (2016). Roles of Fe-S proteins: from cofactor synthesis to iron homeostasis to protein synthesis. Current Opinion in Genetics and Development, 38, 45-51.

[20] Uribarri J and Calvo S. (2013). Dietary phosphorus excess: a risk factor in chronic bone, kidney, and cardiovascular disease? Advances in Nutrition, 4(5), 542-544.

[21] Heaney P, Recker R, Watson P and Lappe M. (2012). Phosphate and carbonate salts of calcium support robust bone building in osteoporosis. American Journal of Clinical Nutrition, 92(1), 101-105.

[22] Garfinkel J, Dilisio F and Agrawal K. (2017). Vitamin D and its effects on articular cartilage and osteoarthritis. The Orthopaedic Journal of Sports Medicine, 5(6), 232.

[23] Kiser D, Golczak M and Palczewski K. (2013). Chemistry of the retinoid (visual) cycle. Chemical Reviews, 114(1), 194-232.

[24] Gilbert C. (2013). The eye signs of vitamin A deficiency. Community Eye Health, 26(84), 66-7.

[25] Morris S. (2012). The role of B vitamins in preventing and treating cognitive impairment and decline. Advances in Nutrition, 3(6), 801-12.

[26] Patel M, Vadlapatla K, Pal D and Mitra K. (2012). Molecular and functional characterization of riboflavin specific transport system in rat brain capillary endothelial cells. Brain Research, 15(1468), 1-10.

[27] Novelli D. (1953). Metabolic functions of pantothenic acid, Physiological reviews, 33(4), 525-543.

[28] Stover J and Field S. (2015). Vitamin B-6. Advances in Nutrition, 6(1), 132-133.

[29] Altun I and Kurutaş B. (2016). Vitamin B complex and vitamin B12 levels after peripheral nerve injury. Neural Regeneration Research, 11(5), 842-845.

[30] Teucher B, Olivares M and Cori H. (2004). Enhancers of iron absorption: Ascorbic acid and other organic acids. International Journal for Vitamin and Nutrition Research, 74(6), 403-419.

[31] Khazai N, Judd E and Tangpricha V. (2008). Calcium and vitamin D: skeletal and extraskeletal health. Current Rheumatology Reports, 10(2), 110-117.

[32] Khan K and Jialal I. (2018) Folic acid (folate) deficiency. In: StatPearls. Treasure Island (FL): StatPearls Publishing; Available from: https://www.ncbi.nlm.nih.gov/books/ NBK535377/

[33] Hershkowitz E, Reshef A, Munich O, Yosefi B and Markel A. (2014). Thiamine deficiency in self-induced refeeding syndrome, an undetected and potentially lethal condition. Case Reports in Medicine, 2014, 6.

[34] Greenblatt J and Delane D. (2018). Zinc supplementation in anorexia nervosa, Journal of Orthomolecular Medicine, 33(1).

[35] De Filippo E, Marra M, Alfinito F, Di Guglielmo M, Majorano P, Cerciello G, De Caprio C, Contaldo F and Pasanisi F. (2016). Hematological complications in anorexia nervosa. European Journal of Clinical Nutrition, 70(11), 13051308. 
[36] EuROFIR Web Services - EuroFIR Food Data, http://www.eurofir.org/food-information/food-compositiondatabases, last accessed on 27 April 2019

[37] Chassy M. (2010). Food safety risks and consumer health. New Biotechnology, 27(5), 534-544.

[38] Simpson K. Shi J, Xue J, Ye X, Jiang Y, Ma Y and Zheng X. (2012). Food biochemistry and food biochemistry. Food Biochemistry and Food Processing: Second Edition. John Wiley \& Sons, Inc. Publication.

[39] Grundy M, Edwards H, Mackie R, Gidley J, Butterworth J and Ellis R. (2016). Re-evaluation of the mechanisms of dietary fibre and implications for macronutrient bioaccessibility, digestion and postprandial metabolism. British Journal of Nutrition, 116(5), 816-833.

[40] Verma V, Rico-Martinez R, Kotra N, King L, Liu J, Snell T and Weber R. (2012). Contribution of water-soluble and insoluble components and their hydrophobic/hydrophilic subfractions to the reactive oxygen species-generating potential of fine ambient aerosols. Environmental Science \& Technology, 46(20), 11384-11392.

[41] Zamindar N, Baghekhandan MS, Nasirpour A and Sheikhzeinoddin M. (2011). Effect of line, soaking and cooking time on water absorption, texture and splitting of red kidney beans. Journal of Food Science and Technology, 50(1), 108-114.

[42] Trailokya A, Srivastava A, Bhole M and Zalte N. (2017). Calcium and calcium salts. Journal of Association of Physicians of India, 65, 100-103.

[43] Matusek A and Wright D. (2010). Ethical dilemmas in treating clients with eating disorders: A review and application of an integrative ethical decision-making model. European Eating Disorders Review, 18(6), 434-452.

\section{How to cite this article}

Popova AT. (2019). Understanding and addressing eating disorders. World Journal of Advanced Research and Reviews, 2(1), 13-21. 